\title{
ON THE COUPLING IMPEDANCE OF A TOROIDAL BEAM PIPE WITH CIRCULAR CROSS SECTION
}

\author{
H. HAHN* \\ Brookhaven National Laboratory, Upton, NY 11973-5000, USA
}

(Received 31 January 1995; in final form 28 August 1995)

\begin{abstract}
Analytic expressions for the non-resonant longitudinal coupling impedance and the centrifugal space charge force in a toroidal beam pipe with circular cross section are presented. The results are obtained by solving Maxwell's equations via a perturbation method in an appropriate orthogonal coordinate system, which is erected in the SerretFrenet frame around the central arc of the torus. General results for the electromagnetic fields due to a tubular beam, which are exact to second order in the inverse curvature radius and are valid in the entire non-resonant region extending well beyond the beam pipe cut off frequency, are obtained. The general results are used to derive a simple expression, valid in the long-wavelength limit, for the centrifugal space charge force on axial particles in a beam with uniform or circular symmetric Gaussian current distribution. The full second-order expression for the curvature-induced residual longitudinal coupling impedance of a filamentary beam is presented from which a simple expansion, valid in the long-wavelength limit, is derived. Numerical estimates confirm that the curvaturedependent non-resonant effects are negligeable for the Relativistic Heavy Ion Collider under construction at this laboratory.
\end{abstract}

KEY WORDS: Coupling impedance, toroidal beam pipe

\section{INTRODUCTION}

In this paper, the topic of coupling impedances and beam-induced forces in a toroidal beam pipe is revisited. In straight, smooth accelerator beam pipe configurations, the space charge forces on a charged test particle due to beam-induced electric and magnetic fields are subject to a near perfect cancellation in the ultra-relativistic limit. It is well known from the studies of effects due to incoherent synchrotron radiation in circular accelerators and later on from the studies of the electron-ring accelerator concept that in toroidal geometries the cancellation is imperfect, resulting in residual, "energy-independent" longitudinal ${ }^{1,2}$ and transverse $e^{3,4}$ forces which can impact the accelerator performance. The subsequent work by Talman ${ }^{5}$ on the "centrifugal space charge force" generated considerable attention to this topic, and it was explored during a dedicated workshop ${ }^{6}$ and in several detailed studies. ${ }^{7-12}$ More recently, Ruggiero's conjecture ${ }^{13}$ of a possibly high longitudinal impedance at cutoff resulting from the curvature in beam pipes revived the interest and lead to the studies by $\mathrm{Ng}$ and

\footnotetext{
${ }^{*}$ Work performed under the auspices of the U.S. Department of Energy.
} 
Warnock ${ }^{14}$ and others, ${ }^{15-16}$ in which the absence of such singularities was proven. Whereas the present-day designs of high-energy hadron accelerators/colliders are based on beam pipes with circular cross section, essentially all theoretical studies assume rectangular geometries, the notable exception being the report by Zotter. ${ }^{17}$ The expressions here presented for the longitudinal coupling impedance and the centrifugal space charge force in curved beam pipes with circular cross section are intended to amend this deficiency.

The original studies of synchrotron radiation, which are obviously of interest in connection with the present work, ignored the impact of metal boundaries. ${ }^{18}$ This treatment is justified by the incoherent character of synchrotron radiation, which is strongest in the vicinity of the critical mode number, well above the beam pipe cutoff frequency, and thus with the particles radiating irrespective of beam dimensions or beam pipe geometry. In order to simplify the problem, similar assumptions were made by Talman ${ }^{5}$ and others ${ }^{9,10}$ in the early studies of the centrifugal space charge force, with the justification that this effect strongly depends on the transverse charge distribution in the beam and less so on the beam pipe geometry.

In contrast to the incoherent radiation by individual particles, the coherent radiation by beam bunches of finite bunch length has a radiation spectrum mainly with wavelengths longer than the dimensions of the bunch, thus located well below the critical mode number and, hence, can be suppressed by metallic shields. ${ }^{19,20}$ It follows that the longitudinal coupling impedance is strongly dependent on the geometry of the shielding environment, as can be seen from the comparison of known $Z / n$ results for different configurations. The expression for the coupling impedance of a completely unshielded beam in free space, orbiting on a circle with radius $R$, has been obtained by Bonch-Osmolovsky from synchrotron radiation results in the long-wavelength limit as ${ }^{21,22}$

$$
Z / n \approx Z_{0} \frac{\Gamma(2 / 3)}{2\left(3 n^{2}\right)^{1 / 3}}(\sqrt{3}+j)
$$

valid for mode numbers $n \ll n_{\text {crit }}=\frac{3}{2} \gamma^{3}$, with $\gamma=\left(1-\beta^{2}\right)^{-1 / 2}$ the normalized energy and $Z_{0}=c \mu_{0}$ in SI-units. This result can be compared to the Pellegrini \& Sessler ${ }^{23,24}$ result for the coupling impedance of parallel plates, valid below cutoff $n_{\mathrm{co}}=\pi R / h$,

$$
Z / n \approx-j Z_{0}\left\{\frac{1}{\beta \gamma^{2}}\left(1+2 \ln \frac{2 h}{\pi a}\right)+\beta\left(\frac{h}{\pi R}\right)^{2}\right\}
$$

where $h$ is the spacing of the plates and $a$ the beam radius. Note that even the character of the result has changed from being radiative, i.e. with losses, to purely capacitive. The more general solution ${ }^{21}$ for this geometry indicates that the residual, $\gamma$-independent, term becomes inductive for $n \sim n_{\text {co }}$ and radiative above cutoff.

The case of a beam circulating between a coaxial pair of infinitely long cylinders was analyzed by Faltens \& Laslett, ${ }^{22}$ with the expression for the residual longitudinal coupling impedance in the ultra-relativistic limit, $\beta \sim 1$, given by

$$
\frac{Z}{n} \sim-j Z_{0}\left(\frac{w}{\pi R}\right)^{2}
$$

valid at mode numbers $n \ll n_{\mathrm{co}}=\frac{1}{2} R / w$, where $2 w$ is the spacing between the cylinders. 
The fields arising from a beam inside a toroidal beam pipe with rectangular cross section of height $h$ and width $2 w$ have been examined by Neil (cited in Ref. 24), with the result for the residual coupling impedance, $\beta \sim 1,\left(n \ll n_{\mathrm{co}}=\pi R / h\right)$

$$
\frac{Z}{n} \approx-j Z_{0}\left(\frac{h}{\pi R}\right)^{2} \sum_{m \text { odd }} \frac{\sinh 2 \xi_{m} w-2 \xi_{m} w}{2 m^{3} \cosh ^{2} \xi_{m} w}
$$

where $\xi_{m}=m \pi / h$. A more general expression, valid for mode numbers up to and beyond cutoff, but below the resonant region, $n \ll(R / w)^{3 / 2}$, was obtained by Hahn $\&$ Tepikian ${ }^{25}$

$$
\frac{Z}{n} \approx-j Z_{0}\left(\frac{h}{\pi R}\right)^{2} \sum_{m \text { odd }}\left[1-3 n^{2}\left(\frac{h}{m \pi R}\right)^{2}\right] \frac{\sinh 2 \xi_{m} w-2 \xi_{m} w}{2 m^{3} \cosh ^{2} \xi_{m} w}
$$

The dependence of the radial force seen by a test particle in a Gaussian bunch in a rectangular beam pipe on the bunch and pipe dimensions was analyzed by Piwinski ${ }^{7}$ and also by Decker. ${ }^{8}$ It will be seen that the results in the present paper for the radial force in a circular symmetric bunch in a beam pipe with circular cross section differ qualitatively due to the absence of quadrupole and higher terms. It follows that, in order to obtain quantitative results in the context of beam instabilities, forces and the concomitant coupling impedances, the beam structure as well as the influence of shielding beam pipes and their geometry must be taken into account.

The obvious reason for considering beam pipes with rectangular cross section in previous studies is the perceived simplicity of the cylindrical versus the unfamiliarity of the toroidal coordinate system. The use of a perturbation treatment, which recently has been successfully applied to the derivation of expressions for the longitudinal coupling impedance in toroidal beam pipes with rectangular cross section, ${ }^{25,26}$ circumvents the mathematical difficulties of the circular cross section and leads, in fact, to simpler analytic results valid as long as the curvature radius is large compared to the beam pipe dimensions.

The results are obtained via the perturbation method developed by Jouguet ${ }^{27}$ for the analysis of the electromagnetic wave propagation in curved waveguides. Using the SerretFrenet frame, an appropriate "local" orthogonal coordinate system $(r, \varphi, \theta)$ can be errected around the central arc of the torus. This choice is preferable to the use of toroidal coordinates, since the local coordinate system reduces to the usual circular-cylinder coordinates $r, \varphi, s=R \theta$ as required for the perturbation treatment of the problem. Whereas the Laplace equation is separable in toroidal coordinates, the vector Helmholtz equation is not, and finding solutions requires approximation treatment in either coordinate system, thereby negating any advantages of the toroidal coordinate system. The metric of this "local" coordinate system and the perturbation method to solve Maxwell's equations are presented in Appendix A.

The primary objective of this paper is the derivation of an expression for the residual longitudinal $Z / n$ of a toroidal beam pipe with circular cross section. The geometry of the toroidal beam pipe is shown in Figure 1, with $b$ representing the beam pipe radius and $R$ the curvature radius of the central arc. The beam is assumed to be filamentary and bunched 


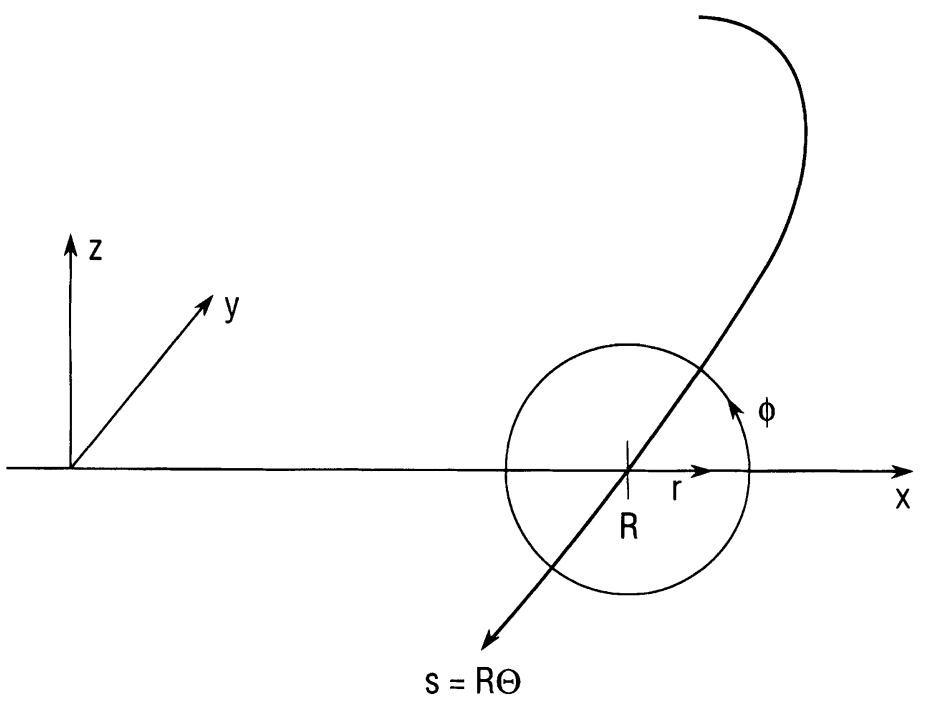

FIGURE 1: Local coordinate system $(r, \varphi, \theta)$ for toroidal beam tube with circular cross section.

with mode number $n$. The result for the residual longitudinal coupling impedance in the ultra-relativistic limit, $\beta \sim 1$, is given by

$$
Z / n \approx-j Z_{0}\left(\frac{b}{2 R}\right)^{2}\left[1-\frac{7}{2} n^{2}\left(\frac{b}{2 R}\right)^{2}\right]
$$

and is valid in the non-resonant region up to $n \ll(R / b)^{3 / 2}$. This result can be directly compared with the above expression (5) for the residual coupling impedance of a rectangular beam pipe which in contrast is given as an infinite series.

The basis for the present study of a toroidal beam pipe is established by first deriving an unabridged version of the well-known expressions for the longitudinal and transverse coupling impedances in a straight beam pipe. Perturbation results to first order in $R^{-1}$, the inverse curvature radius, are then derived, which yield novel expressions for the centrifugal space charge force in a toroidal beam pipe with circular cross section. Second-order perturbation results finally lead to an analytical expression for the residual longitudinal coupling impedance of a toroidal beam pipe. Since wall losses are themselves a small perturbation, they can be ignored in deriving the perturbation results due to the curvature. In the main part of this paper, the toroidal results are given in the low mode number, long-wavelength approximation only, i.e. $n \ll \gamma \cdot R / b \sim \gamma n_{\mathrm{co}}$, which is sufficient for all practical purposes and conveys a better physical picture of the problem. The full perturbation results to first and second order in $R^{-1}$, which are valid in the entire nonresonant region, $n \ll(R / b)^{3 / 2}$, and hence beyond cutoff, are presented in the Appendices B and $\mathrm{C}$ respectively. 


\section{STRAIGHT BEAM PIPE}

The foundation for the present study is laid by first rederiving the well-known expressions for the longitudinal coupling impedance seen by an axial beam, as well as the transverse impedance seen by a radially displaced beam in a straight beam pipe.

In order to prevent a logarithmic divergence of the result for the coupling impedances, the beam must be given a finite transverse size. Solving the case of a tubular beam located at the radius $\rho$ avoids the divergence. This approach corresponds to using the concept of Green's functions without the mathematical formalism and is a necessary step to finding solutions by the perturbation method used in this paper. It is also a convenient way, alternative to the use of potentials, ${ }^{28}$ to finding a solution for a beam with uniform current density inside a radius $a$, as will be demonstrated in the case of the straight beam pipe, for which the results are well known. ${ }^{29}$

The tubular beam is assumed to travel in the $\theta$ direction with velocity $v$ and has the current density

$$
i_{\theta}=J \delta(r-\rho) \cos m \varphi e^{-j n \theta} e^{j \omega t}
$$

with the mode number $n$, wave number $v=n / R$, and circular frequency in natural units $\left(c=1, \mu_{0}=1\right)$

$$
\omega=v \cdot n / R=v v
$$

The associated electromagnetic fields are solutions of the vector Helmholtz equation and are obtained by field matching at $r=\rho$, while imposing Ampère's law on $H_{\varphi}$, and by satisfying the "practical" boundary condition at $r=b$ for a lossy, thick pipe wall, $E_{\theta}=-\mathcal{R}_{s} H_{\varphi}$, with the surface resistance $\mathcal{R}_{s}=\frac{1}{2}(1+j) \omega \delta_{s}, \delta_{s}$ being the skin depth.

The fields induced by the tubular beam centered in a straight beam pipe are transverse magnetic and fully defined by the electric $\theta$ field component. One finds, with the harmonic time dependence suppressed,

- in the inner region $(0<r<\rho)$

$$
E_{\theta i}=j J \frac{\kappa^{2} \rho}{\omega} C_{m}(\kappa \rho) I_{m}(\kappa r) \cos m \varphi e^{-j n \theta}
$$

- and in the outer region $(\rho<r<b)$

$$
E_{\theta o}=j J \frac{\kappa^{2} \rho}{\omega} I_{m}(\kappa \rho) C_{m}(\kappa r) \cos m \varphi e^{-j n \theta}
$$

where

$$
\kappa^{2}=v^{2}-\omega^{2}=\left(1-v^{2}\right) v^{2}=(\nu / \gamma)^{2}
$$

The expressions for the remaining field components are given in Table 1. 
TABLE 1: Field components due to tubular beam in straight pipe.

\begin{tabular}{lccc}
\hline & & $r<\rho$ & $r>\rho$ \\
\hline$E_{r}=-J \frac{\nu \kappa \rho}{\omega} \cos m \varphi e^{-j n \theta}$ & $\times$ & $C_{m}(\kappa \rho) I_{m}^{\prime}(\kappa r) ;$ & $I_{m}(\kappa \rho) C_{m}^{\prime}(\kappa r)$ \\
$E_{\varphi}=+J \frac{m \nu \rho}{\omega r} \sin m \varphi e^{-j n \theta}$ & $\times$ & $C_{m}(\kappa \rho) I_{m}(\kappa r) ;$ & $I_{m}(\kappa \rho) C_{m}(\kappa r)$ \\
$E_{\theta}=j J \frac{\kappa^{2} \rho}{\omega} \cos m \varphi e^{-j n \theta}$ & $\times$ & $C_{m}(\kappa \rho) I_{m}(\kappa r) ;$ & $I_{m}(\kappa \rho) C_{m}(\kappa r)$ \\
$H_{r}=-J \frac{m \rho}{r} \sin m \varphi e^{-j n \theta}$ & $\times$ & $C_{m}(\kappa \rho) I_{m}(\kappa r) ;$ & $I_{m}(\kappa \rho) C_{m}(\kappa r)$ \\
$H_{\varphi}=-J \kappa \rho \cos m \varphi e^{-j n \theta}$ & $\times$ & $C_{m}(\kappa \rho) I_{m}^{\prime}(\kappa r) ;$ & $I_{m}(\kappa \rho) C_{m}^{\prime}(\kappa r)$ \\
$H_{\theta}=0$ & & & \\
\hline
\end{tabular}

The radial dependence is given in terms of modified Bessel functions, $I_{m}(\kappa r)$, and the Bessel function combinations

$$
C_{m}(\kappa r)=K_{m}(\kappa r)-I_{m}(\kappa r) \frac{K_{m}(\kappa b)+j v \gamma \mathcal{R}_{s} K_{m}^{\prime}(\kappa b)}{I_{m}(\kappa b)+j v \gamma \mathcal{R}_{s} I_{m}^{\prime}(\kappa b)}
$$

which for small losses, i.e. $\delta_{s} \ll b$, can be approximated by

$$
C_{m}(\kappa r) \approx K_{m}(\kappa r)-\frac{I_{m}(\kappa r)}{I_{m}(\kappa b)}\left[K_{m}(\kappa b)-(j-1) \frac{v^{2} \gamma^{2} \delta_{s}}{2 b I_{m}(\kappa b)}\right]
$$

where the Wronskian has been used to simplify the expression.

\subsection{Longitudinal coupling impedance in straight beam tube}

The longitudinal coupling impedance is defined in terms of the electric force on an on-axis test particle by

$$
\frac{Z}{n}=-\frac{R}{n I} \int_{0}^{2 \pi}\left(E_{\theta}\right)_{r=0} e^{j n \theta} d \theta
$$

where $I$ is the total peak current of the beam.

Assuming uniform current density inside the beam which has a radius $a$ and is centered in the pipe, one obtains the total on-axis longitudinal electric field component by setting $m=0$ and $J=I d \rho /\left(\pi a^{2}\right)$ in the tubular beam expressions, Table 1 , and by integration of

$$
\left(E_{\theta}\right)_{r=0}=j \frac{I}{\pi a^{2}} \frac{\kappa^{2} e^{-j n \theta}}{\omega} \int_{0}^{a} \rho C_{0}(\kappa \rho) d \rho
$$


The longitudinal coupling impedance follows from Equation (14) as

$$
\frac{Z}{n}=-j \frac{2 R}{v \gamma n a}\left\{\frac{1}{\kappa a}-K_{1}(\kappa a)-\frac{I_{1}(\kappa a) K_{0}(\kappa b)}{I_{0}(\kappa b)}+(j-1) \frac{v^{2} \gamma^{2} \delta_{s}}{2 b} \frac{I_{1}(\kappa a)}{I_{0}^{2}(\kappa b)}\right\}
$$

In the long-wavelength limit, i.e. $\kappa b \ll 1$, which at high energies extends to mode numbers above cutoff $n \ll \gamma R / b \sim \gamma n_{\mathrm{co}}$, this expression simplifies to the well known result ${ }^{30}$ (in SI units with $Z_{0}=c \mu_{0}, v=\beta c$ )

$$
\frac{Z}{n} \approx-j \frac{Z_{0}}{\beta \gamma^{2}}\left(\ln \frac{b}{a}+\frac{1}{2}\right)+(1+j) \beta Z_{0} \frac{\delta_{s}}{2 b}
$$

The coupling impedance of a beam with circular symmetric Gaussian current density is obtained by setting $m=0$ and

$$
J=\frac{I}{2 \pi \sigma^{2}} \exp \left(-\frac{\rho^{2}}{2 \sigma^{2}}\right) d \rho
$$

leading in the long-wavelength limit to $(\sigma \ll b)$

$$
\frac{Z}{n}=-j \frac{Z_{0}}{\beta \gamma^{2}}\left(\ln \frac{b}{\sqrt{2} \sigma}+\frac{C}{2}\right)+(1+j) \beta Z_{0} \frac{\delta_{s}}{2 b}
$$

with Euler's constant $C \approx 0.577$.

\subsection{Transverse impedance of displaced beam in straight beam tube}

A beam of uniform current density inside a radius $a$ which is radially displaced by $\Delta x$ has its boundary given by

$$
r=\Delta x \cos \varphi+\sqrt{a^{2}-(\Delta x \sin \varphi)^{2}}
$$

The forces on the on-axis test particle due to the displacement of the beam can be approximated by considering a tubular beam at $r=a$, having the current density

$$
i_{\theta} \approx \frac{I}{\pi a} \delta(r-a) e^{-j n \theta}\left\{\frac{\Delta x}{a} \cos \varphi-\frac{1}{4}\left(\frac{\Delta x}{a}\right)^{2}(1-\cos 2 \varphi)+\cdots\right\}
$$

To first order in $\Delta x$, only a transverse magnetic dipole field is induced, which results in a radial force on the test particle and is described by the transverse coupling impedance ${ }^{31}$

$$
Z_{T}=j \frac{R}{v I \Delta x} \int_{0}^{2 \pi}(\vec{E}+\vec{v} \times \vec{B})_{r=0} e^{j n \theta} d \theta
$$


Using the above results for a tubular beam, one finds

$$
\left(E_{r}-v H_{\varphi}\right)_{r=0}=-\frac{\kappa}{2 \pi v \gamma^{2} a} C_{1}(\kappa a) I \Delta x
$$

resulting in the expression for the transverse coupling impedance

$$
Z_{T}=-j \frac{\kappa R}{v^{2} \gamma^{2} a}\left\{K_{1}(\kappa a)-I_{1}(\kappa a) \frac{K_{1}(\kappa b)}{I_{1}(\kappa b)}\right\}+(1+j) \frac{\kappa R \delta_{s} I_{1}(\kappa a)}{2 a b I_{1}^{2}(\kappa b)}
$$

which in the long-wavelength limit reduces to the well known expression ${ }^{32}$ (SI units)

$$
Z_{T} \approx-j \frac{Z_{0} R}{\beta^{2} \gamma^{2}}\left(\frac{1}{a^{2}}-\frac{1}{b^{2}}\right)+(1+j) \frac{Z_{0} R \delta_{s}}{b^{3}}
$$

\section{CENTRIFUGAL SPACE CHARGE FORCE}

The electromagnetic fields induced by a tubular beam in a toroidal beam pipe can be derived by a perturbation treatment for which the formalism is developed in the Appendix A and which is valid in the entire non-resonant region, $n \ll(R / b)^{3 / 2}$. Having the solution for the tubular beam allows one, in principle, to find a solution for any current distribution. However, the mathematical manipulations required make it impractical to go beyond a uniform current density beam in the first-order approximation or a filamentary beam for the second-order approximation. This restriction can be relaxed in the long-wavelength limit, and results for the centrifugal space charge force in beams with circular symmetric Gaussian current density have been obtained.

All cases considered start with the solution for the tubular current density which is then given, in local coordinates $r, \varphi, \theta$, by (with the $e^{j \omega t}$ time factor suppressed)

$$
i_{\theta}=J \delta(r-\rho) e^{-j n \theta}
$$

Taking into account the curvature, the charge density follows as

$$
q=\frac{J}{v} \delta(r-\rho)\left(1-\frac{\rho}{R} \cos \varphi\right) e^{-j n \theta}+\frac{1}{R^{2}} \cdots
$$

with $v$ the velocity of the on-axis particle. 
Expanding the fields as a power series in $1 / R$, one can write

$$
\begin{aligned}
E_{r} & =E_{r 0}+\frac{1}{R} E_{r 1}+\frac{1}{R^{2}} \cdots \\
E_{\varphi} & =\frac{1}{R} E_{\varphi 1}+\frac{1}{R^{2}} \cdots \\
E_{\theta} & =j \tilde{E}_{\theta 0}+j \frac{1}{R} \tilde{E}_{\theta 1}+\frac{1}{R^{2}} \cdots \\
H_{r} & =\frac{1}{R} H_{r 1}+\frac{1}{R^{2}} \cdots \\
H_{\varphi} & =H_{\varphi 0}+\frac{1}{R} H_{\varphi 1}+\frac{1}{R^{2}} \cdots \\
H_{\theta} & =j \frac{1}{R} \tilde{H}_{\theta 1}+\frac{1}{R^{2}} \cdots
\end{aligned}
$$

The zeroth-order terms are obtained from the straight beam pipe solution as follows - in the inner region, $0<r<\rho$

$$
\begin{aligned}
E_{r 0 i} & =-J \frac{\nu \kappa \rho}{\omega} C_{0}(\kappa \rho) I_{1}(\kappa r) \\
\tilde{E}_{\theta 0 i} & =J \frac{\kappa^{2} \rho}{\omega} C_{0}(\kappa \rho) I_{0}(\kappa r) \\
H_{\varphi 0 i} & =-J \kappa \rho C_{0}(\kappa \rho) I_{1}(\kappa r)
\end{aligned}
$$

- in the outer region, $\rho<r<b$

$$
\begin{aligned}
E_{r 0 o} & =-J \frac{\nu \kappa \rho}{\omega} I_{0}(\kappa \rho) C_{0}^{\prime}(\kappa r) \\
\tilde{E}_{\theta 0 o} & =J \frac{\kappa^{2} \rho}{\omega} I_{0}(\kappa \rho) C_{0}(\kappa r) \\
H_{\varphi 0 o} & =-J \kappa \rho I_{0}(\kappa \rho) C_{0}^{\prime}(\kappa r)
\end{aligned}
$$

As shown in Appendix A, the first-order perturbation terms $\tilde{E}_{\theta 1}$ and $\tilde{H}_{\theta 1}$ must satisfy the differential equations

$$
\Delta_{T} \tilde{E}_{\theta 1}-\kappa^{2} \tilde{E}_{\theta 1}=-\Delta_{T}\left(r \cos \varphi \tilde{E}_{\theta 0}\right)+\frac{1}{r} \frac{\partial}{\partial r}\left\{r^{2} \cos \varphi\left(\nu E_{r 0}+\omega H_{\varphi 0}\right)\right\}
$$


and

$$
\Delta_{T} \tilde{H}_{\theta 1}-\kappa^{2} \tilde{H}_{\theta 1}=-\Delta_{T}\left(r \cos \varphi \tilde{H}_{\theta 0}\right)-\sin \varphi\left(\omega E_{r 0}+v H_{\varphi 0}\right)
$$

with the transverse Laplace operator

$$
\Delta_{T} u=\frac{1}{r} \frac{\partial}{\partial r}\left(r \frac{\partial u}{\partial r}\right)+\frac{1}{r^{2}} \frac{\partial^{2} u}{\partial \varphi^{2}}
$$

In addition, the perturbation terms must satisfy the boundary condition $E_{\theta 1 o}=0$ and $E_{\varphi 1 o}=0$ at $r=b$ on the beam pipe, from now on assumed lossless, and they must satisfy the continuity conditions at $r=\rho$ for $E_{\theta 1}, E_{\varphi 1}, H_{\theta 1}$ and $H_{\varphi 1}$.

Exact first-order results for the curvature-induced fields of a tubular beam centered around the beam pipe axis have been obtained by aid of the symbolic manipulation program MACSYMA and are recorded in Appendix B.

\subsection{Long-wavelength approximation}

The general expressions for the curvature-induced transverse space charge force in Appendix B are somewhat opaque, and more informative results are found by considering the long-wavelength approximation, $\kappa b \ll 1$ or $n \ll \gamma R / b$. The long-wavelength expressions for the field perturbations of a tubular beam in the inner region, $r<\rho$, to first order in $1 / R$ are listed in Table 2 for use in the derivation of the centrifugal space charge force expressions.

It is seen that the curvature induced fields have, in first order, pure dipole character, i.e. only a $1 \varphi$ dependence. Although not analyzed in this paper, one easily sees that a radially displaced beam would induce quadrupole fields, i.e. $2 \varphi$ dependence. The presence of sextupole fields, ${ }^{6,7}$ i.e. $3 \varphi$ dependence, in a first-order approximation, is only possible in non-circular beams or beam pipes. In the typical hadron accelerator/collider with essentially equal horizontal and vertical emittances, the effects due to the non-circular beam geometry will average out in first approximation, thereby justifying the simplifications made here.

The curvature-induced fields subsist in the ultra-relativistic limit and give rise to a centrifugal space charge force on a test particle. Inside of a tubular beam, $r<\rho$, one finds in the ultra-relativistic limit, i.e. $v \sim c$,

$$
E_{r 1 i}-v H_{\varphi 1 i}=\frac{J \rho}{2}\left(2 \ln \frac{b}{\rho}+1-\frac{\rho^{2}}{b^{2}}\right) \cos \varphi
$$

The centrifugal space charge force on an on-axis test particle inside a tubular beam can be defined by an equivalent radial electric field as follows (SI units)

$$
F_{x}=\frac{Z_{0} I}{2 \pi R}\left(\ln \frac{b}{\rho}+\frac{1}{2}-\frac{1}{2} \frac{\rho^{2}}{b^{2}}\right)
$$


TABLE 2: First order field perturbations in long-wavelength limit inside a tubular beam

$$
\begin{aligned}
& E_{r 1 i}=J \frac{\rho}{16 v}\left\{\left[8-4 v^{2}\left(2 \rho^{2}-r^{2}\right)\right] \ln \frac{b}{\rho}\right. \\
& \left.+4\left(1-\frac{\rho^{2}}{b^{2}}\right)+v^{2}\left[7 b^{2}-6 \rho^{2}+2 r^{2}-\frac{\rho^{2}}{b^{2}}\left(\rho^{2}+2 r^{2}\right)\right]\right\} \cos \varphi \\
& E_{\varphi 1 i}=-J \frac{\rho}{16 v}\left\{\left[8-4 v^{2}\left(2 \rho^{2}+r^{2}\right)\right] \ln \frac{b}{\rho}\right. \\
& \left.+4\left(1-\frac{\rho^{2}}{b^{2}}\right)+v^{2}\left[7 b^{2}-6 \rho^{2}-2 r^{2}-\frac{\rho^{2}}{b^{2}}\left(\rho^{2}-2 r^{2}\right)\right]\right\} \sin \varphi \\
& E_{\theta 1 i}=-J \frac{v r \rho}{2 v}\left\{1-\frac{\rho^{2}}{b^{2}}+2 \ln \frac{b}{\rho}\right\} \cos \varphi \\
& H_{r 1 i}=-J \frac{\rho}{16}\left\{\left[8+4 v^{2}\left(2 \rho^{2}+r^{2}\right)\right] \ln \frac{b}{\rho}\right. \\
& \left.+4\left(1-\frac{\rho^{2}}{b^{2}}\right)-v^{2}\left[7 b^{2}-6 \rho^{2}-2 r^{2}-\frac{\rho^{2}}{b^{2}}\left(\rho^{2}-2 r^{2}\right)\right]\right\} \sin \varphi \\
& H_{\varphi 1 i}=-J \frac{\rho}{16}\left\{\left[8+4 v^{2}\left(2 \rho^{2}-r^{2}\right)\right] \ln \frac{b}{\rho}\right. \\
& \left.+4\left(1-\frac{\rho^{2}}{b^{2}}\right)-v^{2}\left[7 b^{2}-6 \rho^{2}+2 r^{2}-\frac{\rho^{2}}{b^{2}}\left(\rho^{2}+2 r^{2}\right)\right]\right\} \cos \varphi \\
& H_{\theta 1 i}=J \frac{v r \rho}{2}\left\{1-\frac{\rho^{2}}{b^{2}}+2 \ln \frac{b}{\rho}\right\} \sin \varphi
\end{aligned}
$$

In the case of a beam with uniform current density, the equivalent radial electric field is given by

$$
F_{x}=\frac{Z_{0} I}{2 \pi a^{2} R} \int_{0}^{a}\left(2 \ln \frac{b}{\rho}+1-\frac{\rho^{2}}{b^{2}}\right) \rho d \rho
$$

which after integration yields

$$
F_{x}=\frac{Z_{0} I}{2 \pi R}\left(\ln \frac{b}{a}+1-\frac{1}{4} \frac{a^{2}}{b^{2}}\right)
$$


In the case of a beam with circular symmetric Gaussian current density, one finds for the equivalent radial electric field

$$
F_{x}=\frac{Z_{0} I}{2 \pi R}\left(\ln \frac{b}{2 \sigma}+\frac{1+C}{2}-\frac{1}{2} \frac{\sigma^{2}}{b^{2}}\right)
$$

with $C$ the Euler constant. The results for uniform and Gaussian beams are numerically comparable to the simpler tubular geometry if as representative dimension $\rho=a$, respectively $\rho=2 \sigma$ is taken. The force field on off-axis particles is still dipolar, albeit non linear, i.e. $r$-dependent, but a more detailed discussion of this effect is outside the scope of this paper.

Numerical estimates prove the centrifugal space charge force to be a negligible effect for the Relativistic Heavy Ion Collider under construction at this laboratory. In the worst case in RHIC, the peak current is $I \approx 10 \mathrm{~A}$, the average transverse beam size $a \approx 2 \mathrm{~mm}$, which with $R=243 \mathrm{~m}$ and $b=35 \mathrm{~mm}$ results in $F_{x} \approx 10 \mathrm{~V} / \mathrm{m}$ as compared to the dipole magnet strength at injection of $1.2 \times 10^{8} \mathrm{~V} / \mathrm{m}$.

\section{RESIDUAL COUPLING IMPEDANCE}

In this section, the residual longitudinal coupling impedance of a toroidal beam pipe to an on-axis filamentary beam will be derived. Since this effect must be independent of the direction of curvature, an expansion to second order in $1 / R$ is required. It is known from previous studies that the residual coupling impedance does not exhibit a logarithmic divergence, if the transverse beam size is reduced to zero. Consequently, the study can be limited to filamentary beams.

A full second-order treatment has been carried out, and the results for the curvatureinduced coupling impedance of a filamentary beam in the non-resonant region, but valid at all beam velocities, are recorded in Appendix C. However, the lengthy equations involving Bessel functions are tedious to manage even with the help of MACSYMA. Limiting the solution to ultra-relativistic particles loses only terms in $\left(\gamma^{2} R^{2}\right)^{-1}$ and leads to considerable simplifications and greater transparency of the results.

The second-order field perturbations in $E_{\theta}$ are solutions of the differential equation for modified Bessel functions with a forcing term

$$
\Delta_{T} \tilde{E}_{\theta 2}-\kappa^{2} \tilde{E}_{\theta 2}=-\Delta_{T}\left(r \cos \varphi \tilde{E}_{\theta 1}\right)+\frac{1}{r} \frac{\partial}{\partial r}\left\{r^{2} \cos \varphi\left(\nu E_{r 1}+\omega H_{\varphi 1}\right)\right\}
$$

The first-order perturbations can be written as

$$
\begin{aligned}
\tilde{E}_{\theta 1} & =\hat{e}_{\theta 1} \cos \varphi \\
E_{r 1} & =\hat{e}_{r 1} \cos \varphi \\
H_{\varphi 1} & =\hat{h}_{\varphi 1} \cos \varphi
\end{aligned}
$$


In the ultra-relativistic case, these expressions for an on-axis filamentary beam become (i.e. $\kappa \rightarrow 0, \rho \rightarrow 0$ )

$$
\begin{aligned}
& \hat{e}_{\theta 1}=-\frac{I}{2 \pi} v r \ln \frac{b}{r} \\
& \dot{e}_{r 1}=-\frac{I}{2 \pi}\left\{\frac{1}{2}-\frac{1}{2} \ln \frac{b}{r}-\frac{v^{2} b^{2}}{16}\left(7-\frac{r^{2}}{b^{2}}+4 \frac{r^{2}}{b^{2}} \ln \frac{b}{r}\right)\right\} \\
& \hat{h}_{\varphi 1}=-\frac{I}{2 \pi}\left\{\frac{1}{2}+\frac{1}{2} \ln \frac{b}{r}-\frac{\nu^{2} b^{2}}{16}\left(7-\frac{r^{2}}{b^{2}}+r \frac{4^{2}}{b^{2}} \ln \frac{b}{r}\right)\right\}
\end{aligned}
$$

The second-order field perturbations of an on-axis filamentary beam are excited by firstorder perturbations with pure dipole, i.e. $1 \varphi$-dependence. The factor $\cos ^{2} \varphi=\frac{1}{2}+\frac{1}{2} \cos 2 \varphi$ in the forcing term implies that the second-order fields have $\varphi$ independent and quadrupole, i.e. $2 \varphi$, dependent terms, hence

$$
\tilde{E}_{\theta 2}=\tilde{e}_{\theta 2}+\hat{e}_{\theta 2} \cos 2 \varphi
$$

Only the $\varphi$-independent term $\tilde{e}_{\theta 2}$ leads to an on-axis electric field component in $\theta$-direction which is responsible for the residual coupling impedance. The differential Equation (39) thus reduces for the relevant $\varphi$-independent $\tilde{e}_{\theta 2}$ component in the ultra-relativistic limit, i.e. $\kappa=0$, to

$$
\frac{1}{r} \frac{\partial}{\partial r}\left(r \frac{\partial \tilde{e}_{\theta 2}}{\partial r}\right)=-\frac{1}{2 r} \frac{\partial}{\partial r}\left(r \frac{\partial r \hat{e}_{\theta 1}}{\partial r}\right)+\frac{\nu}{2 r} \frac{\partial}{\partial r}\left(r^{2}\left(\hat{e}_{r 1}+\hat{h}_{\varphi 1}\right)\right)
$$

Using the above expressions, (41)-(43), for an ultra-relativistic filamentary beam, the differential equation for $\tilde{e}_{\theta 2}$ now takes the form

$$
\frac{1}{r} \frac{\partial}{\partial r}\left(r \frac{\partial \tilde{e}_{\theta 2}}{\partial r}\right)=-v \frac{I}{2 \pi}\left\{3-2 \ln \frac{b}{r}-v^{2} b^{2}\left(\frac{7}{8}-\frac{r^{2}}{2 b^{2}}+\frac{r^{2}}{b^{2}} \ln \frac{b}{r}\right)\right\}
$$

together with the boundary conditions on the beam pipe wall

$$
\left(\tilde{e}_{\theta 2}\right)_{r=b}=0
$$

and, replacing the matching condition,

$$
\left(\frac{\partial \tilde{e}_{\theta 2}}{\partial r}\right)_{r=0}=0
$$

The solution is found to be

$$
\tilde{\boldsymbol{e}}_{\theta 2}=\frac{I}{2 \pi} \frac{v b^{2}}{4}\left\{\left(1-\frac{7}{8} v^{2} b^{2}\right)\left(1-\frac{r^{2}}{b^{2}}\right)+2\left(1+\frac{\nu^{2} r^{2}}{8}\right) \frac{r^{2}}{b^{2}} \ln \frac{b}{r}\right\}
$$


from which follow the expressions for the on-axis field

$$
\left(\tilde{e}_{\theta 2}\right)_{r=0}=\nu \frac{I}{2 \pi} \frac{b^{2}}{4}\left(1-\frac{7}{8} \nu^{2} b^{2}\right)
$$

and for the residual longitudinal coupling impedance seen by a filamentary beam in the ultra-relativistic case, $\gamma \rightarrow \infty$,

$$
\frac{Z}{n}=-j \frac{b^{2}}{4 R^{2}}\left(1-\frac{7}{8} v^{2} b^{2}\right)
$$

The full second-order expression for the curvature-induced longitudinal coupling impedance valid in the non-resonant region, $n \ll(R / b)^{3 / 2}$, is given in Appendix $\mathrm{C}$ as follows

$$
\begin{aligned}
\frac{Z}{n}= & -\frac{j}{v v^{2} R^{2}}\left[\frac{3}{8 I_{0}^{2}(\kappa b)}\left\{1-I_{0}^{2}(\kappa b)\right\}\right. \\
& -\frac{v^{2}}{4 \kappa^{2} I_{0}^{2}(\kappa b) I_{1}^{\prime}(\kappa b)}\left\{2 \kappa^{2} b^{2} I_{0}(\kappa b)-\left[3 \kappa^{2} b^{2}-4\left(1-I_{0}^{2}(\kappa b)\right)\right] I_{1}^{\prime}(\kappa b)\right\} \\
& +\frac{v^{4}}{24 \kappa^{4} I_{0}^{2}(\kappa b) I_{1}(\kappa b) I_{1}^{\prime}(\kappa b)}\left\{12 \kappa^{3} b^{3} I_{0}^{2}(\kappa b)-15 \kappa^{3} b^{3} I_{0}(\kappa b) I_{1}^{\prime}(\kappa b)\right. \\
& \left.\left.-2\left[5 \kappa^{2} b^{2}-8\left(1-I_{0}^{2}(\kappa b)\right)\right] I_{1}(\kappa b) I_{1}^{\prime}(\kappa b)\right\}\right]
\end{aligned}
$$

Expansion with respect to $\kappa=v / \gamma$ yields the curvature-induced coupling impedance term in the long-wavelength limit, $n \ll \gamma R / b$, which together with the impedance due to space charge and wall losses in Equation (17) yield the total impedance of a toroidal beam pipe with circular cross section (SI units)

$$
\begin{aligned}
\frac{Z}{n} \approx & -j \frac{Z_{0}}{\beta \gamma^{2}}\left(\ln \frac{b}{a}+\frac{1}{2}\right)+(1+j) \beta Z_{0} \frac{\delta_{s}}{2 b} \\
& -j \beta Z_{0} \frac{b^{2}}{4 R^{2}}\left(1-\frac{7}{8} v^{2} b^{2}\right)+j \frac{Z_{0}}{16 \beta \gamma^{2}} \frac{b^{2}}{R^{2}}\left(1-2 v^{2} b^{2}+\frac{109}{48} v^{4} b^{4}\right)
\end{aligned}
$$

Taking the RHIC geometry for a numerical example confirms that the curvature contribution to the coupling impedance is negligible in the non-resonant region. 


\section{REFERENCES}

1. V.K. Neil, Ph.D. Dissertation (U. California, 1960).

2. V.K. Neil, D.L. Judd and L.J. Laslett, Rev. Sci. Instrum., 32, 267 (1961).

3. D.W. Kerst, Lawrence Berkeley Laboratory Report LBID-533/BETA-13 (1981).

4. L.J. Laslett, Lawrence Berkeley Laboratory Report LBID-562/BETA-14 (1981).

5. R. Talman, Phys. Rev. Letters, 56, 1429 (1986).

6. E. Keil, Report CERN LEP-TH/85-40 (1985).

7. A. Piwinski, Report CERN/LEP-TH/85-43 (1985).

8. G.A. Decker, Ph.D. Dissertation (Cornell University, 1986).

9. M. Bassetti and D. Brandt, Report CERN/LEP-TH/86-04 (1986).

10. M. Bassetti, Report CERN/LEP-TH/86-13 (1986).

11. P.J. Channell, Los Alamos National Laboratory Reports AT-6: ATN-86-15 (1986), AT-6: ATN-86-30 (1986).

12. E.P. Lee, Particle Accelerators, 25, 241 (1990).

13. A.G. Ruggiero, Brookhaven National Laboratory Reports AD/RHIC-AP-56 and AD/AP-TN-10 (1988).

14. K.-Y. Ng and R. Warnock, Proc. 1989 Particle Accelerator Conf., Chicago, IL, p. 798; Phys. Rev. D., 40, 231 (1989).

15. R.L. Warnock and P. Morton, Particle Accelerators, 25, 133 (1990).

16. H. Hahn and S. Tepikian, Proc. 2nd European Particle Accelerator Conf., Nice (Editions Frontières 1990) p. 1043.

17. B. Zotter, Report CERN/ISR-TH/77-56 (1977).

18. J. Schwinger, Phys. Rev., 70, 798 (1946); Phys Rev., 75, 1912 (1949).

19. L.I. Schiff, Rev. Sci. Instr., 17, 6 (1946).

20. J.S. Nodvick and D.S. Saxon, Phys. Rev., 96, 180 (1954).

21. A.G. Bonch-Osmolovsky, JINR Dubna Report P9-6318 (1972) (Lawrence Livermore Laboratory, UCRLTrans-1480).

22. A. Faltens and L.J. Laslett, Particle Accelerators, 4, 151 (1973); Proc. 1975 ISABELLE Summer Study, Brookhaven National Laboratory, Report BNL 20550, vol. II, p. 486.

23. C. Pellegrini and A. Sessler, CERN Report ISR-TH/67-40; Proc. Symposium Electron Ring Accelerators, p. 442, (1968) (Lawrence Radiation Laboratories Report UCRL-18103); Nuovo Cimento, 3A, 116 (1971).

24. C. Pellegrini and A. Sessler, Nuovo Cimento, 53B, 198 (1968).

25. H. Hahn and S. Tepikian, Proc. 1991 IEEE Particle Accelerator Conf., San Francisco, CA, p. 1707.

26. H. Hahn, S. Tepikian and G. Dôme, Particle Accelerators, 49, 163 (1995).

27. M. Jouguet, Cables \& Transmissions, 1, 133 (1947).

28. J.-L. Laclare, Proc. 1984 CERN Accelerator School, Report CERN 85-19, p. 377.

29. King-Yuen Ng, Physics of Particle Accelerators, AIP Conference Proceedings \#184, M. Month and M. Dienes, eds., (Am. Inst. Phys., New York, 1989), Vol. 1, p. 472.

30. V. Kelvin Neil and Andrew M. Sessler, Rev. Sci. Instr., 36, 429 (1965).

31. F. Sacherer, Proc. 9th Intern. Conf. High Energy Accelerators, Stanford, CA, p. 347 (1974).

32. L. Jackson Laslett, V. Kelvin Neil and Andrew M. Sessler, Rev. Sci. Instr., 36, 436 (1965).

33. L. Lewin, Theory of Waveguides, Chapter 4.4 (J. Wiley \& Sons, New York 1975).

34. W.K.H. Panofsky and W.A. Wenzel, Rev. Sci. Instr., 27, 967 (1956). 


\section{APPENDIX A PERTURBATIVE SOLUTION OF MAXWELL'S EQUATIONS IN THE LOCAL COORDINATE SYSTEM}

Using the Serret-Frenet frame, an orthogonal curvilinear coordinate system appropriate to the toroidal beam pipe geometry can be errected around the central arc of the torus. The Cartesian coordinates $x, y, z$ are found in terms of the local coordinates $r, \varphi, \theta$ by

$$
\begin{aligned}
& x=(R+r \cos \varphi) \cos \theta \\
& y=-(R+r \cos \varphi) \sin \theta \\
& z=r \sin \varphi
\end{aligned}
$$

The metric coefficients are defined by the line element

$$
d l^{2}=d r^{2}+r^{2} d \varphi^{2}+(R+r \cos \varphi)^{2} d \theta^{2}
$$

as follows

$$
g_{r}=1, g_{\varphi}=r, g_{\theta}=R g
$$

with

$$
g=1+\frac{r}{R} \cos \varphi .
$$

Assuming time harmonic fields of the general form

$$
\mathcal{F}(r, \varphi) e^{-j n \theta} e^{j \omega t}
$$

with $\omega=v v$ and $v=n / R$, one can write Maxwell's equations in the source free regions as

$$
\begin{aligned}
\frac{\partial g \mathcal{E}_{\theta}}{r \partial \varphi}+j \nu \mathcal{E}_{\varphi} & =-j \omega g \mathcal{H}_{r} \\
j \nu \mathcal{E}_{r}+\frac{\partial g \mathcal{E}_{\theta}}{\partial r} & =j \omega g \mathcal{H}_{\varphi} \\
\frac{\partial r \mathcal{E}_{\varphi}}{r \partial r}-\frac{\partial \mathcal{E}_{r}}{r \partial \varphi} & =-j \omega \mathcal{H}_{\theta} \\
\frac{\partial g \mathcal{H}_{\theta}}{r \partial \varphi}+j \nu \mathcal{H}_{\varphi} & =j \omega g \mathcal{E}_{r} \\
j \nu \mathcal{H}_{r}+\frac{\partial g \mathcal{H}_{\theta}}{\partial r} & =-j \omega g \mathcal{E}_{\varphi} \\
\frac{\partial r \mathcal{H}_{\varphi}}{r \partial r}-\frac{\partial \mathcal{H}_{r}}{r \partial \varphi} & =j \omega \mathcal{E}_{\theta}
\end{aligned}
$$


Perturbative solutions can be found by expanding the fields in inverse powers of the curvature radius, or explicitly

$$
\begin{aligned}
\mathcal{E}_{r, \varphi} & =E_{r, \varphi}+\frac{1}{R} e_{r, \varphi}+\cdots \\
\mathcal{E}_{\theta} & =j\left(\tilde{E}_{\theta}+\frac{1}{R} \tilde{e}_{\theta}+\cdots\right) \\
\mathcal{H}_{r, \varphi} & =H_{r, \varphi}+\frac{1}{R} h_{r, \varphi}+\cdots \\
\mathcal{H}_{\theta} & =j\left(\tilde{H}_{\theta}+\frac{1}{R} \tilde{h}_{\theta}+\cdots\right)
\end{aligned}
$$

The resulting expressions for Maxwell's equations to first order in $R^{-1}$ are given as follows

$$
\begin{aligned}
\frac{1}{r} \frac{\partial \tilde{e}_{\theta}}{\partial \varphi}+v e_{\varphi}+\omega h_{r} & =-\frac{\partial}{\partial \varphi}\left(\cos \varphi \tilde{E}_{\theta}\right)-\omega r \cos \varphi H_{r} \\
\nu e_{r}+\frac{\partial \tilde{e}_{\theta}}{\partial r}-\omega h_{\varphi} & =-\cos \varphi \frac{\partial}{\partial r}\left(r \tilde{E}_{\theta}\right)+\omega r \cos \varphi H_{\varphi} \\
\frac{\partial}{\partial r}\left(r e_{\varphi}\right)-\frac{\partial e_{r}}{\partial \varphi}-\omega r \tilde{h}_{\theta} & =0 \\
\frac{1}{r} \frac{\partial \tilde{h}_{\theta}}{\partial \varphi}+\nu h_{\varphi}-\omega e_{r} & =-\frac{\partial}{\partial \varphi}\left(\cos \varphi \tilde{H}_{\theta}\right)+\omega r \cos \varphi E_{r} \\
\nu h_{r}+\frac{\partial \tilde{h}_{\theta}}{\partial r}+\omega e_{\varphi} & =-\cos \varphi \frac{\partial}{\partial r}\left(r \tilde{H}_{\theta}\right)-\omega r \cos \varphi E_{\varphi} \\
& \frac{\partial}{\partial r}\left(r h_{\varphi}\right)-\frac{\partial h_{r}}{\partial \varphi}+\omega r \tilde{e}_{\theta}=0
\end{aligned}
$$

Decoupling Maxwell's equations and reducing them to two independent partial differential equations in the azimuthal components $\tilde{e}_{\theta}$ and $\tilde{h}_{\theta}$ can be achieved by introducing complex transverse fields ${ }^{33}$

$$
\mathcal{E}_{T}=\mathcal{E}_{r}+i \mathcal{E}_{\varphi}
$$

and

$$
\mathcal{H}_{T}=\mathcal{H}_{r}+i \mathcal{H}_{\varphi}
$$

and the differential operators

$$
\mathrm{D} \equiv \frac{\partial}{\partial r}+i \frac{\partial}{r \partial \varphi} ; \mathrm{D}^{*} \equiv \frac{\partial}{\partial r}-i \frac{\partial}{r \partial \varphi}
$$


The perturbation formulation of Maxwell's equations now takes the form

$$
\begin{aligned}
\mathrm{D} \tilde{e}_{\theta}+v e_{T}+i \omega h_{T} & =-\mathrm{D}\left(r \cos \varphi \tilde{E}_{\theta}\right)-i \omega r \cos \varphi H_{T} \\
\operatorname{ReD}^{*}\left(i r e_{T}\right)-\omega r \tilde{h}_{\theta} & =0 \\
\mathrm{D} \tilde{h}_{\theta}+\nu h_{T}-i \omega e_{T} & =-\mathrm{D}\left(r \cos \varphi \tilde{H}_{\theta}\right)+i \omega r \cos \varphi E_{T} \\
\operatorname{ReD}^{*}\left(i r h_{T}\right)-\omega r \tilde{e}_{\theta} & =0
\end{aligned}
$$

Whereas the real part of the following operator combination vanishes

$$
\operatorname{ReD}^{*} i r \mathrm{D} \mathcal{F}_{\theta} \equiv 0
$$

elimination of the transversu nerd components has become possible, resulting in the uncoupled differential equations

$$
\begin{aligned}
\operatorname{ReD}^{*}\left(r \mathrm{D} \tilde{e}_{\theta}\right)-\kappa^{2} r \tilde{e}_{\theta}= & -\operatorname{ReD}^{*} r \mathrm{D} r \cos \varphi \tilde{E}_{\theta} \\
& +\nu \operatorname{ReD}^{*} r^{2} \cos \varphi E_{T}-\omega \operatorname{ReD}^{*} i r^{2} \cos \varphi H_{T} \\
\operatorname{ReD}^{*}\left(r \mathrm{D} \tilde{h}_{\theta}\right)-\kappa^{2} r \tilde{h}_{\theta}= & -\operatorname{ReD}^{*} r \mathrm{D} r \cos \varphi \tilde{H}_{\theta} \\
& +\nu \operatorname{ReD}^{*} r^{2} \cos \varphi H_{T}+\omega \operatorname{ReD}^{*} i r^{2} \cos \varphi E_{T}
\end{aligned}
$$

with $\kappa^{2}=v^{2}-\omega^{2}=v^{2} / \gamma^{2}$. Furthermore, it is easily verified that the operator combination represents

$$
\operatorname{ReD}^{*} r \mathrm{D} \mathcal{F}_{\theta} \equiv r \Delta_{T} \mathcal{F}_{\theta}
$$

with the transverse Laplacian operator

$$
\Delta_{T}=\frac{1}{r} \frac{\partial}{\partial r}\left(r \frac{\partial}{\partial r}\right)+\frac{1}{r^{2}} \frac{\partial^{2}}{\partial \varphi^{2}}
$$

After some manipulations, one finds the independent differential equations for the azimuthal perturbations 


$$
\begin{aligned}
\Delta_{T} \tilde{e}_{\theta}-\kappa^{2} \tilde{e}_{\theta}= & -\Delta_{T}\left(r \cos \varphi \tilde{E}_{\theta}\right) \\
& +\frac{1}{r} \frac{\partial}{\partial r}\left\{r^{2} \cos \varphi\left(\nu E_{r}+\omega H_{\varphi}\right)\right\} \\
& +\frac{\partial}{\partial \varphi}\left\{\cos \varphi\left(\nu E_{\varphi}-\omega H_{r}\right)\right\} \\
\Delta_{T} \tilde{h}_{\theta}-\kappa^{2} \tilde{h}_{\theta}= & -\Delta_{T}\left(r \cos \varphi \tilde{H}_{\theta}\right) \\
& +\frac{1}{r} \frac{\partial}{\partial r}\left\{r^{2} \cos \varphi\left(\nu H_{r}-\omega E_{\varphi}\right)\right\} \\
& +\frac{\partial}{\partial \varphi}\left\{\cos \varphi\left(\nu H_{\varphi}+\omega E_{r}\right)\right\}
\end{aligned}
$$

Having determined $\tilde{e}_{\theta}$ and $\tilde{h}_{\theta}$, the remaining transverse components can be obtained as follows

$$
\begin{aligned}
\kappa^{2} e_{r}= & -v \frac{\partial \tilde{e}_{\theta}}{\partial r}-v \cos \varphi \frac{\partial r \tilde{E}_{\theta}}{\partial r}+\omega^{2} r \cos \varphi E_{r} \\
& -\frac{\omega}{r} \frac{\partial \tilde{h}_{\theta}}{\partial \varphi}-\omega \frac{\partial}{\partial \varphi} \cos \varphi \tilde{H}_{\theta}+\omega \nu r \cos \varphi H_{\varphi} \\
\kappa^{2} h_{r}= & -v \frac{\partial \tilde{h}_{\theta}}{\partial r}-v \cos \varphi \frac{\partial r \tilde{H}_{\theta}}{\partial r}+\omega^{2} r \cos \varphi H_{r} \\
& +\frac{\omega}{r} \frac{\partial \tilde{e}_{\theta}}{\partial \varphi}+\omega \frac{\partial}{\partial \varphi} \cos \varphi \tilde{E}_{\theta}-\omega \nu r \cos \varphi E_{\varphi} \\
\kappa^{2} e_{\varphi}= & -\frac{v}{r} \frac{\partial \tilde{e}_{\theta}}{\partial \varphi}-\nu \frac{\partial}{\partial \varphi} \cos \varphi \tilde{E}_{\theta}+\omega^{2} r \cos \varphi E_{\varphi} \\
& +\omega \frac{\partial \tilde{h}_{\theta}}{\partial r}+\omega \cos \varphi \frac{\partial r \tilde{H}_{\theta}}{\partial r}-\omega \nu r \cos \varphi H_{r} \\
\kappa^{2} h_{\varphi}= & -\frac{v}{r} \frac{\partial \tilde{h}_{\theta}}{\partial \varphi}-v \frac{\partial}{\partial \varphi} \cos \varphi \tilde{H}_{\theta}+\omega^{2} r \cos \varphi H_{\varphi} \\
& -\omega \frac{\partial \tilde{e}_{\theta}}{\partial r}-\omega \cos \varphi \frac{\partial r \tilde{E}_{\theta}}{\partial r}+\omega \nu r \cos \varphi E_{r}
\end{aligned}
$$

The solution is uniquely determined by imposing boundary and continuity conditions. 
The internal consistency of the results can be checked by testing for a divergence free solution using $\operatorname{div} \vec{E}=0$ :

$$
\frac{\partial r e_{r}}{\partial r}+\frac{\partial e_{\varphi}}{\partial \varphi}+v r \tilde{e}_{\theta}=-r \cos \varphi\left(E_{r}+\frac{\partial r E_{r}}{\partial r}\right)-r \frac{\partial \cos \varphi E_{\varphi}}{\partial \varphi}
$$

and $\operatorname{div} \vec{H}=0$ :

$$
\frac{\partial r h_{r}}{\partial r}+\frac{\partial h_{\varphi}}{\partial \varphi}+v r \tilde{h}_{\theta}=-r \cos \varphi\left(H_{r}+\frac{\partial r H_{r}}{\partial r}\right)-r \frac{\partial \cos \varphi H_{\varphi}}{\partial \varphi}
$$

The curvature-induced transverse force on a test charge can be expressed by an equivalent electric field with the components

$$
F_{r}=\frac{1}{R}\left(e_{r}-v h_{\varphi}\right)=-\frac{\partial \tilde{e}_{\theta}}{v R \partial r}-\cos \varphi \frac{\partial r \tilde{E}_{\theta}}{v R \partial r}+\frac{v r}{R} \cos \varphi H_{\varphi}
$$

and

$$
F_{\varphi}=\frac{1}{R}\left(e_{\varphi}+v h_{r}\right)=-\frac{\partial \tilde{e}_{\theta}}{\nu R r \partial \varphi}-\frac{\partial \cos \varphi \tilde{E}_{\theta}}{\nu R \partial \varphi}-\frac{v r}{R} \cos \varphi H_{r}
$$

It is noted that the Panofsky-Wenzel theorem ${ }^{34}$ is not directly applicable to toroidal structures.

\section{APPENDIX B FIRST-ORDER PERTURBATION SOLUTION}

In this appendix, the unabridged first-order solution for the fields due to a tubular beam in a toroidal beam pipe, assumed lossless with circular cross section, is recorded. The perturbation treatment used is valid for all beam energies, but is limited to the non-resonance region, $n \ll(R / b)^{3 / 2}$.

The current density in the beam is taken to be

$$
i_{\theta}=J \delta(r-\rho) e^{-j n \theta} e^{j \omega t}
$$

The associated zeroth-order solution is

- in the inner region $(0<r<\rho)$

$$
\begin{aligned}
E_{r 0 i} & =-J \frac{\nu \kappa \rho}{\omega} C_{0}(\kappa \rho) I_{1}(\kappa r) \\
\tilde{E}_{\theta 0 i} & =J \frac{\kappa^{2} \rho}{\omega} C_{0}(\kappa \rho) I_{0}(\kappa r) \\
H_{\varphi 0 i} & =-J \kappa \rho C_{0}(\kappa \rho) I_{1}(\kappa r)
\end{aligned}
$$


- and in the outer region $(\rho<r<b)$

$$
\begin{aligned}
E_{r 0 o} & =-J \frac{\nu \kappa \rho}{\omega} I_{0}(\kappa \rho) C_{0}^{\prime}(\kappa r) \\
\tilde{E}_{\theta 0 o} & =J \frac{\kappa^{2} x}{\omega} I_{0}(\kappa \rho) C_{0}(\kappa r) \\
H_{\varphi 0 o} & =-J \kappa \rho I_{0}(\kappa \rho) C_{0}^{\prime}(\kappa r)
\end{aligned}
$$

with the common factor $e^{-j n \theta} e^{j \omega t}$ suppressed and the symbols as defined in the main section of this paper, but in view of the lossless pipe walls,

$$
C_{0}(\kappa r)=K_{0}(\kappa r)-I_{0}(\kappa r) \frac{K_{0}(\kappa b)}{I_{0}(\kappa b)}
$$

Using the interactive symbolic manipulation program MACSYMA, the following results for the first-order perturbations were obtained:

- in the inner region, $0<r<\rho$,

$$
\begin{aligned}
\tilde{E}_{\theta 1 i}=J \frac{\kappa \rho}{2 \omega} C_{0}(\kappa \rho) & \left\{2 I_{1}(\kappa r)-\kappa r I_{0}(\kappa r)\right. \\
& \left.-\frac{v^{2}}{\kappa^{2}}\left[2 \kappa r I_{0}(\kappa r)-\left(4-\kappa^{2} r^{2}\right) I_{1}(\kappa r)\right]-Q_{i}^{E} I_{1}(\kappa r)\right\} \cos \varphi
\end{aligned}
$$

with

$$
\begin{aligned}
& Q_{i}^{E}=\frac{1}{C_{0}(\kappa \rho) I_{0}(\kappa b) I_{1}(\kappa b)}\{\left\{\frac{v^{2} b}{\kappa}\left(1-\frac{\rho^{2}}{b^{2}}\right) I_{0}(\kappa \rho)+\frac{\rho}{b} I_{1}(\kappa \rho)\right. \\
&+ {\left[\left(2+4 \frac{v^{2}}{\kappa^{2}}-v^{2} \rho^{2}\right) C_{0}(\kappa \rho)\right.} \\
&\left.\left.+\kappa \rho C_{0}^{\prime}(\kappa \rho)\right] I_{0}(\kappa b) I_{1}(\kappa b)\right\}
\end{aligned}
$$

and

$$
\tilde{H}_{\theta 1 i}=J \frac{\nu \rho}{\kappa} C_{0}(\kappa \rho)\left\{\kappa r I_{0}(\kappa r)+Q_{i}^{H} I_{1}(\kappa r)\right\} \sin \varphi
$$

with

$$
Q_{i}^{H}=\frac{b I_{0}(\kappa \rho)+\rho I_{0}(\kappa b)\left[C_{1}(\kappa \rho) I_{1}(\kappa b)+\kappa b C_{0}^{\prime}(\kappa \rho) I_{0}(\kappa b)\right]}{b C_{0}(\kappa \rho) I_{0}(\kappa b) I_{1}^{\prime}(\kappa b)}
$$


- in the outer region, $\rho<r<b$,

$$
\begin{aligned}
\tilde{E}_{\theta 1 o}= & -J \frac{\nu \rho}{2 \omega} I_{0}(\kappa \rho)\left\{\nu b \frac{I_{1}(\kappa r)}{I_{0}(\kappa b) I_{1}(\kappa b)}\right. \\
& +\nu r\left(2+\frac{\kappa^{2}}{\nu^{2}}\right) C_{0}(\kappa r)+\kappa \nu r^{2} C_{0}^{\prime}(\kappa r) \\
& \left.+\frac{\kappa}{v}\left(\nu^{2} \rho^{2}-\kappa \rho \frac{I_{1}(\kappa \rho)}{I_{0}(\kappa \rho)}\right) C_{1}(\kappa r)\right\} \cos \varphi \\
\tilde{H}_{\theta 1 o}= & J \frac{\nu \rho}{\kappa} \frac{I_{0}(\kappa \rho)}{I_{0}(\kappa b) I_{1}^{\prime}(\kappa b)} \\
& \left\{I_{1}(\kappa r)+\kappa r I_{0}(\kappa b) I_{1}^{\prime}(\kappa b) C_{0}(\kappa r)\right. \\
& \left.+\frac{\rho}{b} \frac{I_{1}(\kappa \rho) I_{0}(\kappa b)}{I_{0}(\kappa \rho)}\left[I_{1}(\kappa b) C_{1}(\kappa r)+\kappa b I_{0}(\kappa b) C_{0}^{\prime}(\kappa r)\right]\right\} \sin \varphi
\end{aligned}
$$

The curvature-induced transverse force on a test charge within the tubular beam can be expressed in terms of an equivalent electric field with the components

$$
\begin{aligned}
F_{r}= & -J \frac{\kappa^{2} \rho}{2 \nu \omega R} C_{0}(\kappa \rho)\left\{3 I_{0}(\kappa r)-\frac{1}{\kappa r}\left(2+\kappa^{2} r^{2}\right) I_{1}(\kappa r)\right. \\
& +\frac{\nu^{2}}{\kappa^{2}}\left[\left(2-\kappa^{2} r^{2}\right) I_{0}(\kappa r)-\frac{1}{\kappa r}\left(4+\kappa^{2} r^{2}\right) I_{1}(\kappa r)\right] \\
& \left.-Q_{i}^{E}\left(I_{0}(\kappa r)-\frac{1}{\kappa r} I_{1}(\kappa r)\right)\right\} \cos \varphi
\end{aligned}
$$

and

$$
\begin{gathered}
F_{\varphi}=J \frac{\kappa^{2} \rho}{2 \nu \omega R} C_{0}(\kappa \rho)\left\{I_{0}(\kappa r)+2 \frac{I_{1}(\kappa r)}{\kappa r}-Q_{i}^{E} \frac{I_{1}(\kappa r)}{\kappa r}\right. \\
\left.-\frac{v^{2}}{\kappa^{2}}\left[2 I_{0}(\kappa r)-\left(4-\kappa^{2} r^{2}\right) \frac{I_{1}(\kappa r)}{\kappa r}\right]\right\} \sin \varphi
\end{gathered}
$$

Of special interest is the radial on-axis component,

$$
\left(F_{r}\right)_{r=0}=-J \frac{\kappa^{2} \rho}{2 \nu \omega R} C_{0}(\kappa \rho)\left\{2-\frac{1}{2} Q_{i}^{E}\right\} \cos \varphi
$$




\section{APPENDIX C SECOND-ORDER PERTURBATION SOLUTION}

In the limit of an on-axis filamentary beam, the $\varphi$-independent second-order perturbation of the azimuthal electric field component, which is responsible for the residual longitudinal coupling impedance, is a solution of the modified Bessel differential equation of order zero with forcing term

$$
\begin{aligned}
\frac{1}{r} \frac{d}{d r}\left(r \frac{d \tilde{e}_{\theta 2}}{d r}\right)-\kappa^{2} \tilde{e}_{\theta 2}= & \frac{I}{2 \pi \omega}\left\{P_{00} I_{0}(\kappa r)+P_{20} \kappa^{2} r^{2} I_{0}(\kappa r)\right. \\
& +P_{11} \kappa r I_{1}(\kappa r)+P_{31} \kappa^{3} r^{3} I_{1}(\kappa r) \\
& +Q_{00} K_{0}(\kappa r)+Q_{20} \kappa^{2} r^{2} K_{0}(\kappa r) \\
& \left.+Q_{11} \kappa r K_{1}(\kappa r)+Q_{31} \kappa^{3} r^{3} K_{1}(\kappa r)\right\}
\end{aligned}
$$

Using MACSYMA, the $P$ and $Q$ factors in the above expressions were found to be

$$
\begin{aligned}
P_{00}= & -\frac{3}{2} \frac{K_{0}(\kappa b)}{I_{0}(\kappa b)}+\frac{\nu^{2}}{4 \kappa^{2}} \frac{4 \kappa b I_{0}(\kappa b)-\left[3 \kappa b+8 I_{1}(\kappa b) K_{0}(\kappa b)\right] I_{1}^{\prime}(\kappa b)}{I_{0}(\kappa b) I_{1}(\kappa b) I_{1}^{\prime}(\kappa b)} \\
& +\frac{\nu^{4}}{2 \kappa^{4}} \frac{\kappa b I_{0}(\kappa b)-3 I_{1}(\kappa b)}{I_{0}(\kappa b) I_{1}(\kappa b) I_{1}^{\prime}(\kappa b)} \\
P_{20}= & -\frac{9 v^{2}}{4 \kappa^{2}} \frac{K_{0}(\kappa b)}{I_{0}(\kappa b)}-\frac{3 v^{4}}{2 \kappa^{4}} \frac{K_{0}(\kappa b)}{I_{0}(\kappa b)} \\
P_{11}= & -\frac{3}{4} \frac{K_{0}(\kappa b)}{I_{0}(\kappa b)}-\frac{11 v^{2}}{2 \kappa^{2}} \frac{K_{0}(\kappa b)}{I_{0}(\kappa b)}+\frac{\nu^{4}}{2 \kappa^{4}} \frac{\kappa b+2 I_{1}(\kappa b) K_{0}(\kappa b)}{I_{0}(\kappa b) I_{1}(\kappa b)} \\
P_{31}= & -\frac{v^{2}}{2 \kappa^{2}} \frac{K_{0}(\kappa b)}{I_{0}(\kappa b)} \\
Q_{00}= & \frac{3}{2}+\frac{2 v^{2}}{\kappa^{2}} \\
Q_{20}= & \frac{9 v^{2}}{4 \kappa^{2}}+\frac{3 v^{4}}{2 \kappa^{4}} \\
Q_{11}= & -\frac{3}{4}-\frac{11 v^{2}}{2 \kappa^{2}}+\frac{v^{4}}{\kappa^{4}} \\
Q_{31}= & -\frac{v^{4}}{2 \kappa^{4}}
\end{aligned}
$$



form

Together with the boundary condition $\tilde{e}_{\theta 2}^{\prime}(0)=0$, one finds the solution in its general

$$
\begin{aligned}
\tilde{e}_{\theta 2}(\kappa r)=\frac{I}{2 \pi \omega}\{ & \frac{1}{2} P_{00} \kappa r I_{1}(\kappa r) \\
& -\frac{1}{6} P_{20}\left[\kappa^{2} r^{2} I_{0}(\kappa r)-\kappa r\left(2+\kappa^{2} r^{2}\right) I_{1}(\kappa r)\right] \\
& +\frac{1}{4} P_{11}\left[\kappa^{2} r^{2} I_{0}(\kappa r)-2 \kappa r I_{1}(\kappa r)\right] \\
& +\frac{1}{24} P_{31}\left[\kappa^{2} r^{2}\left(8+3 \kappa^{2} r^{2}\right) I_{0}(\kappa r)-\kappa r\left(16+8 \kappa^{2} r^{2}\right) I_{1}(\kappa r)\right] \\
& -\frac{1}{2} Q_{00} \kappa r K_{1}(\kappa r) \\
& -\frac{1}{6} Q_{20}\left[\kappa^{2} r^{2} K_{0}(\kappa r)+\kappa r\left(2+\kappa^{2} r^{2}\right) K_{1}(\kappa r)\right] \\
& -\frac{1}{4} Q_{11}\left[\kappa^{2} r^{2} K_{0}(\kappa r)+2 \kappa r K_{1}(\kappa r)\right] \\
& -\frac{1}{24} Q_{31}\left[\kappa^{2} r^{2}\left(8+3 \kappa^{2} r^{2}\right) K_{0}(\kappa r)+\kappa r\left(16+8 \kappa^{2} r^{2}\right) K_{1}(\kappa r)\right] \\
+ & \left.P I_{0}(\kappa r)\right\}
\end{aligned}
$$

where $P$ remains to be determined by the boundary condition at $r=b, \tilde{e}_{\theta 2}(\kappa b)=0$ whence

$$
\begin{aligned}
P= & \frac{3}{8 I_{0}^{2}(\kappa b)}-\frac{\nu^{2}}{4 \kappa^{2}} \frac{2 \kappa^{2} b^{2} I_{0}(\kappa b)+\left(4-3 \kappa^{2} b^{2}\right) I_{1}^{\prime}(\kappa b)}{I_{0}^{2}(\kappa b) I_{1}^{\prime}(\kappa b)} \\
& -\frac{\nu^{4}}{24 \kappa^{4}} \frac{3 \kappa^{3} b^{3} I_{0}^{2}(\kappa b)-5 \kappa^{2} b^{2} I_{0}(\kappa b) I_{1}(\kappa b)-10 \kappa b I_{1}^{2}(\kappa b)-16 I_{1}(\kappa b) I_{1}^{\prime}(\kappa b)}{I_{0}^{2}(\kappa b) I_{1}(\kappa b) I_{1}^{\prime}(\kappa b)}
\end{aligned}
$$
by

The curvature induced coupling impedance seen by a filamentary on-axis beam is defined

$$
\frac{Z}{n}=-j \frac{2 \pi}{\nu R^{2} I} \tilde{e}_{\theta 2}(0)
$$


resulting in the unabridged expression (natural units)

$$
\begin{aligned}
\frac{Z}{n}= & -\frac{j}{v \nu^{2} R^{2}}\left[\frac{3}{8 I_{0}^{2}(\kappa b)}\left\{1-I_{0}^{2}(\kappa b)\right\}\right. \\
& -\frac{v^{2}}{4 \kappa^{2} I_{0}^{2}(\kappa b) I_{1}^{\prime}(\kappa b)}\left\{2 \kappa^{2} b^{2} I_{0}(\kappa b)-\left[3 \kappa^{2} b^{2}-4\left(1-I_{0}^{2}(\kappa b)\right) I_{1}^{\prime}(\kappa b)\right\}\right. \\
& +\frac{v^{4}}{24 \kappa^{4} I_{0}^{2}(\kappa b) I_{1}(\kappa b) I_{1}^{\prime}(\kappa b)}\left\{12 \kappa^{3} b^{3} I_{0}^{2}(\kappa b)-15 \kappa^{3} b^{3} I_{0}(\kappa b) I_{1}^{\prime}(\kappa b)\right. \\
& \left.\left.-2\left[5 \kappa^{2} b^{2}-8\left(1-I_{0}^{2}(\kappa b)\right)\right] I_{1}(\kappa b) I_{1}^{\prime}(\kappa b)\right\}\right]
\end{aligned}
$$

which is valid in the non-resonant region for all energies. 